\title{
Evaluation of Inoculation Methods for Screening Horseradish Cultivars for Resistance to Verticillium dahliae
}

\author{
N. Atibalentja and D. M. Eastburn, Department of Crop Sciences, University of Illinois, Urbana 61801
}

\begin{abstract}
Atibalentja, N., and Eastburn, D. M. 1997. Evaluation of inoculation methods for screening horseradish cultivars for resistance to Verticillium dahliae. Plant Dis. 81:356-362.

Four inoculation methods - colonized oatseed, root dip, infested soil, and set dip-were tested in the greenhouse for their effectiveness in identifying horseradish cultivars that are resistant to Verticillium wilt of horseradish. Examination of the inoculum density-disease relationships derived with each of these methods on susceptible (647A) and resistant (769A) cultivars showed that all were effective, though at varying degrees, in differentiating between susceptible and resistant reactions. Results were more consistent with the root dip method as it produced the largest least-squares means difference in wilt index between the two cultivars, the highest $r^{2}$, the lowest coefficient of variation, the shortest incubation periods, and the highest incidence of foliar symptoms. Overall, inoculum concentrations accounted for only a small amount of the total variation in wilt index $\left(0.14 \leq r^{2} \leq 0.73\right)$. This observation, in accord with previous reports on other hosts of Verticillium dahliae, would suggest that inoculum densities may not be a good predictor of the severity of Verticillium wilt of horseradish.
\end{abstract}

Verticillium wilt of horseradish, $A r$ moracia rusticana P. Gaertn., B. Mey. \& Scherb., has become a serious threat to the horseradish industry in the United States $(13,19,22)$. Yield losses result from the discoloration and deterioration of the root, the marketable part of the plant, following colonization by Verticillium dahliae Kleb. alone or in conjunction with secondary invaders. These include bacteria such as Pseudomonas spp., and fungi such as Fusarium spp. (22).

Root symptoms are classified as "pepper," "peg," and "rot" $(13,14)$. "Pepper," the most common symptom, is characterized by the presence of dark brown to black vascular discoloration. "Peg" refers to isolated portions of the root that have become hard and dry in texture, brown in color, and often are walled off from the rest of the root. "Rot," the most severe symptom of the disease, applies to roots with internal areas that have begun to decompose. Foliar symptoms may or may not develop. When they appear, they start from the lower leaves and proceed upward. Affected leaves first exhibit chlorosis followed by necrosis. Symptoms often start near the leaf margins and progress inward

Corresponding author: D. M. Eastburn

E-mail: eastburn@uiuc.edu

Portion of a dissertation submitted by the first author in partial fulfillment of the requirements for the degree of Master of Science in Plant Pathology.

Accepted for publication 20 December 1996.

Publication no. D-1997-0218-04R

(C) 1997 The American Phytopathological Society along the veins, resulting in a characteristic V-shaped lesion. Symptoms commonly develop on one half of a leaf. A cross section of the petiole reveals a blackening of the vascular tissue from which the pathogen can be isolated.

There is, at present, no single method available to effectively control the disease. Soil fumigation and fungicide treatment of planting stock failed to provide acceptable levels of protection (19; D. M. Eastburn, unpublished). Crop rotation, as practiced now by Illinois horseradish growers, may reduce the amount of initial inoculum in the soil; however, this method alone is of limited value due to the pathogen's extended host range (8) and ability to survive for long periods of time in the absence of susceptible hosts (30). Even with reduced inoculum densities (IDs), the pathogen may still cause significant damage when susceptible cultivars are grown. On cotton, Gossypium hirsutum L., 20 to $50 \%$ infection occurred with IDs as low as 0.3 to 1 microsclerotia per $\mathrm{g}$ of soil, and an ID of 3.5 microsclerotia per $\mathrm{g}$ of soil resulted in $100 \%$ infection (4). On tomato, Lycopersisclerotia per $\mathrm{g}$ of soil were sufficient to cause $100 \%$ infection (15). con esculentum Mill., IDs of 4.5 microcultivars for resistance to Verticillium dahliae

${ }^{a}$ Grams of $V$. dahliae colonized oatseed per liter of soil.

${ }^{c}$ Number of $V$. dahliae microsclerotia per gram of soil.
Disease resistance has the potential to reduce crop losses, with a minimum effort by growers, in an environmentally safe, cost-effective manner. In addition, resistance can be combined with other control measures to achieve optimal levels of disease management. Identification of sources of resistance, however, depends on suitable screening techniques, including an effective method of inoculation $(16,27,32)$.

Numerous inoculation methods have been reported by investigators working with Verticillium spp. on horseradish (8, $13,19,22)$ or other hosts $(18,23,26,28,29$, $31)$. However, there is currently no data to suggest which of these methods would be most appropriate for the screening of horseradish cultivars for resistance to $V$. dahliae. The purpose of this study was to evaluate the effectiveness of four inoculation methods to identify sources of resistance to Verticillium wilt of horseradish.

\section{MATERIALS AND METHODS}

Four inoculation methods, each consisting of five inoculum concentrations and a control (Table 1), were tested in the greenhouse for their effectiveness in resolving susceptible and resistance reactions to Verticillium wilt of horseradish. Cultivars 647A and 769A, susceptible and resistant (based on observations in growers' fields), respectively, were used.

Experimental design. The three factors (cultivar, inoculation method, and inoculum concentration) were combined into a 2 $\times 4 \times 6$ cross-nested factorial, in which inoculum concentrations were nested within methods, which in turn were crossed with cultivars. The resulting treatments were arranged in a completely randomized design with four replications; the experimental unit consisted of a single plant in a 15-cm-diameter clay pot.

Root cuttings (sets), $2 \mathrm{~cm}$ diameter by 8 $\mathrm{cm}$ long, were used as planting materials. Sets were washed under tap water, dipped in $70 \%$ ethanol for $15 \mathrm{~s}$, soaked in $0.5 \%$ sodium hypochlorite $(\mathrm{NaOCl})$ for $10 \mathrm{~min}$,

Table 1. Inoculation methods and inoculum concentrations tested for the screening of horseradish

\begin{tabular}{lrrrrr}
\hline Inoculation method & \multicolumn{4}{c}{ Inoculum concentrations } \\
\hline Colonized oatseed & $5^{\mathrm{a}}$ & 10 & 15 & 20 & 25 \\
Root dip & $10^{\mathrm{b}}$ & 50 & 100 & 500 & 1,000 \\
Infested soil & $10^{\mathrm{c}}$ & 50 & 100 & 500 & 1,000 \\
Set dip & $10^{\mathrm{b}}$ & 50 & 100 & 500 & 1,000 \\
\hline
\end{tabular}

${ }^{\mathrm{b}}$ Number of $V$. dahliae microsclerotia per milliliter of inoculum suspension. 
rinsed in tap water, and blotted dry prior to inoculation.

Inoculum preparation and inoculation procedure. Verticillium inoculum consisted of a $1: 1$ mixture $(\mathrm{vol} / \mathrm{vol})$ of two isolates, HR001 and HR015, both from horseradish (13). The preparation of the colonized oatseed inoculum was based upon slight modifications of a procedure previously reported (13). Whole oatseed (200 g per liter of distilled water) was soaked for $16 \mathrm{~h}$ in 2-liter Erlenmeyer flasks and autoclaved at $121^{\circ} \mathrm{C}$ for $1 \mathrm{~h}$. After the excess water was poured off, the oatseed was incubated at room temperature (22 to $24^{\circ} \mathrm{C}$ ) for $24 \mathrm{~h}$ and then autoclaved again for $1 \mathrm{~h}$. The contents of each flask were subsequently inoculated with 5 plugs $(0.5$
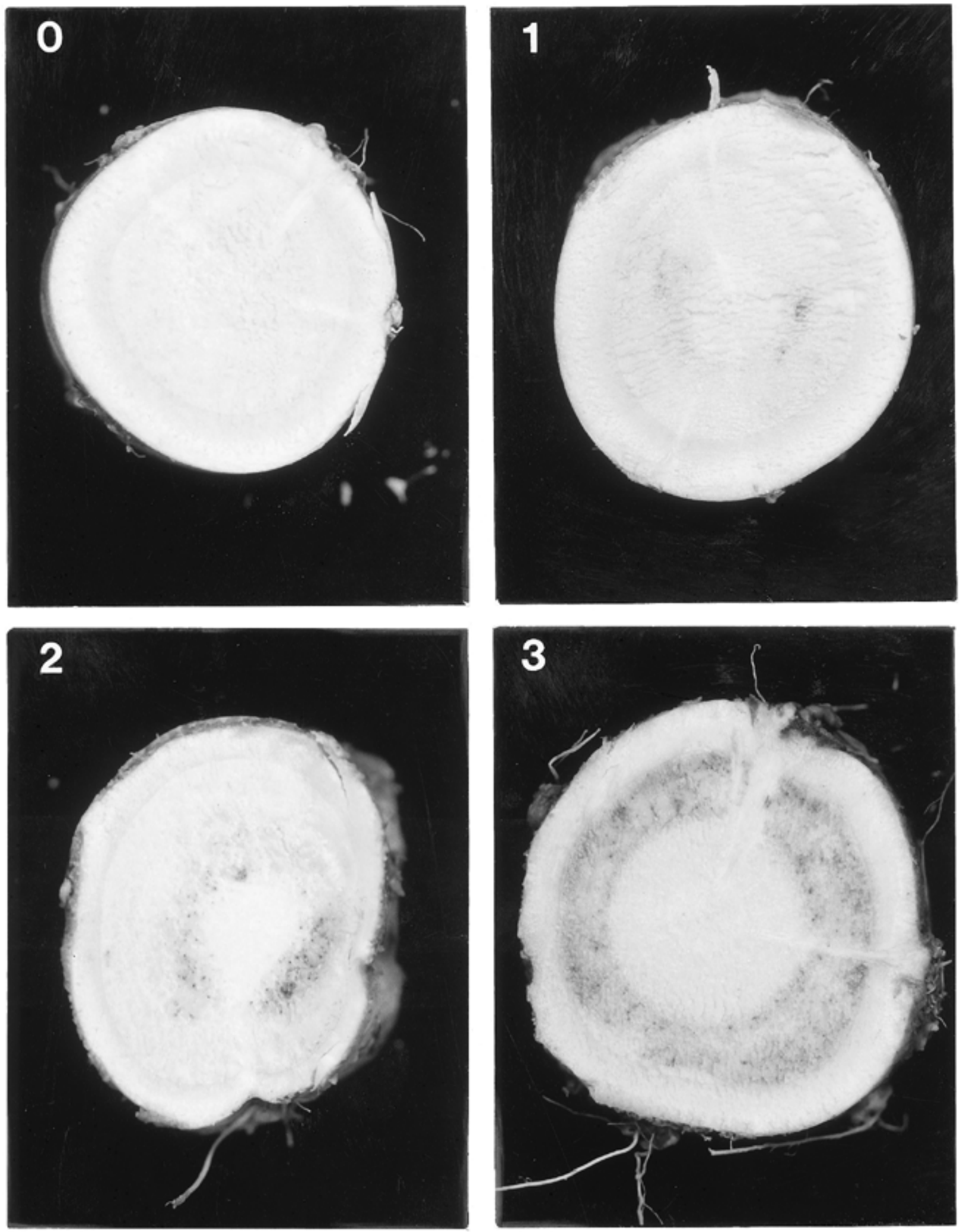

Fig. 1. The 0 to 3 pepper symptom rating scale used to evaluate the severity of vascular discoloration of horseradish caused by Verticillium dahliae: $0=$ clean (no vascular discoloration); $1=$ low (trace to less than $10 \%$ of the root cross section with vascular discoloration); $2=$ moderate (10 to $50 \%$ of the root cross section with vascular discoloration); and $3=$ high (more than $50 \%$ of the root cross section with vascular discoloration). 
$\mathrm{cm}$ diameter) cut from a 1-month-old potato-dextrose agar culture of $V$. dahliae. The flasks were incubated for 4 weeks at room temperature, with shaking to prevent oat grains from clumping. The oatseed was then air dried under a fume hood for 4 days and mixed with appropriate amounts of steam-sterilized potting mix (1:1:1, soil/ peat/perlite), hereafter designated as standard soil. Finally, surface-sterilized sets

Table 2. Selected statistics from the covariance analysis of wilt index on two horseradish cultivars, 647A and 769A, inoculated with Verticillium dahliae during two greenhouse trials involving four inoculation methods ${ }^{\mathrm{a}}$

\begin{tabular}{lccc}
\hline Inoculation method & $\boldsymbol{r}^{\mathbf{2}}$ & Coefficient of variation $\mathbf{( \% )}$ & Difference $^{\mathbf{b}}$ \\
\hline Colonized oatseed & 0.46 & 47 & 1.2 \\
Root dip & 0.73 & 34 & 1.7 \\
Infested soil & 0.31 & 59 & 0.9 \\
Set dip & 0.58 & 40 & 1.2
\end{tabular}

${ }^{\mathrm{a}}$ Each method included five inoculum concentrations, and the analysis was based on pooled data from the two trials.

${ }^{\mathrm{b}}$ Least-squares means difference between the susceptible (647A) and the resistant (769A) cultivars.

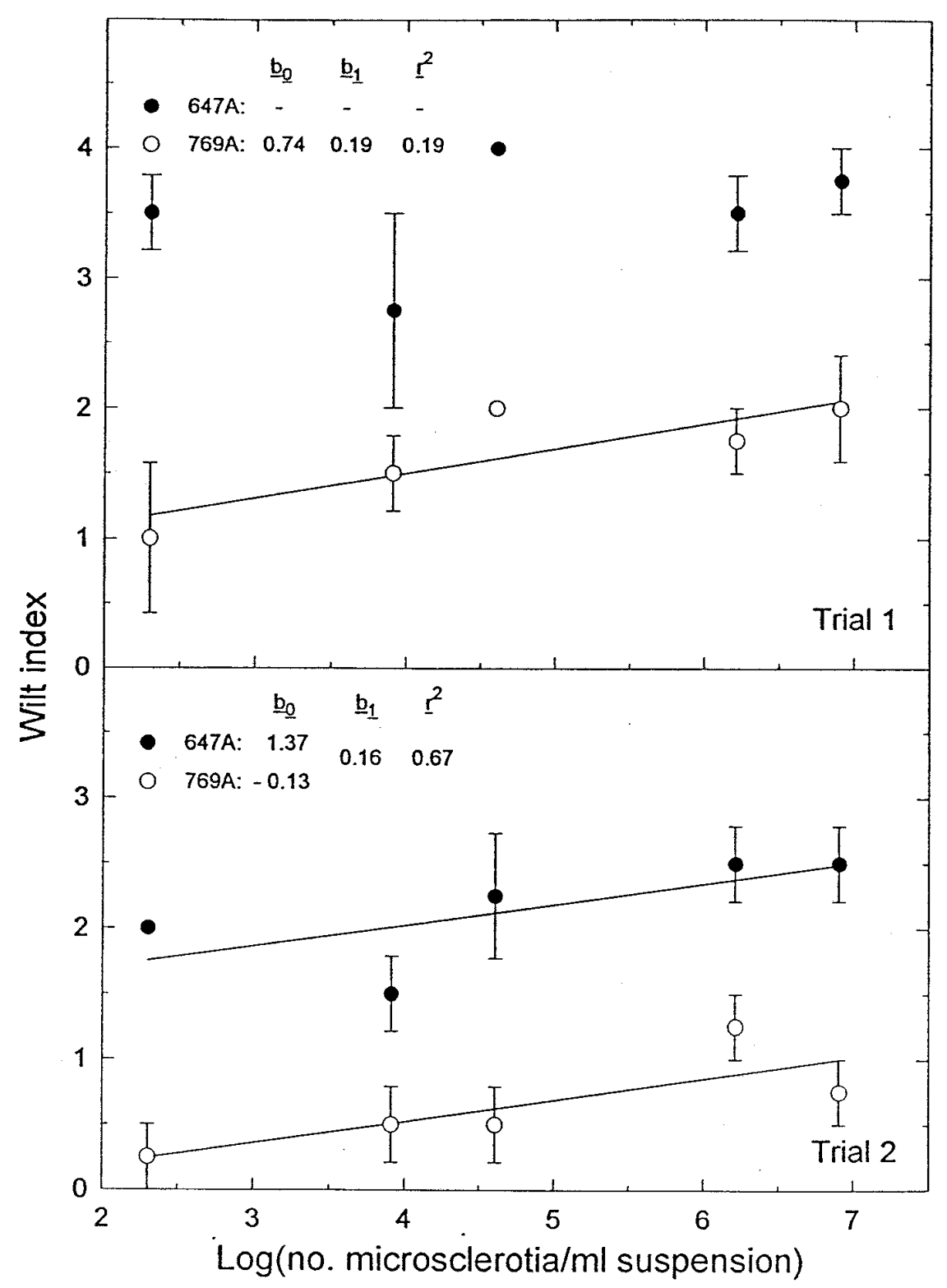

Fig. 2. Effect of inoculum concentrations of Verticillium dahliae on wilt index of two horseradish cultivars inoculated by root dip method. Each observation represents mean of four replications; vertical bars represent standard errors. Wilt index is on 0 to 4 scale, with $0=$ neither foliar nor pepper symptoms, and $4=$ plant exhibiting foliar symptoms and $>50 \%$ of root cross section with vascular discoloration. Trial 2 : parameters are from covariance analysis of combined data for the two cultivars.

were planted in the infested soil. Controls were planted in noninfested standard soil.

Stock suspensions of microsclerotia used in the remaining three inoculation methods were prepared following a procedure similar to that of Chang and Eastburn (8). Six plates $(100 \times 15 \mathrm{~mm})$ of 1 -monthold potato-dextrose agar cultures of $\mathrm{V}$. dahliae were blended with $200 \mathrm{ml}$ of distilled water for $1 \mathrm{~min}$ at high speed in a Waring Blender (Dynamics Corporation of America, New Hartford, CT). The resulting suspension was passed through a nested series of 500-, 180-, and 38- $\mu \mathrm{m}$ sieves. Only the materials (mostly microsclerotia) on the $38-\mu \mathrm{m}$ sieve were collected, rinsed with running tap water, and resuspended in distilled water. The concentration of the stock suspension was estimated by counting the number of microsclerotia with a hemacytometer.

For the root and set dip methods, the final microsclerotial concentrations were achieved by diluting the stock suspension with sterile, distilled water, into which $0.5 \%$ agar had been melted to give the inoculum suspension a thick consistency (26, 28,29). In the set dip method, surfacesterilized sets were immersed in the inoculum suspension for approximately $15 \mathrm{~s}$ and planted immediately in standard soil. In the root dip method, surface-sterilized sets were first allowed to sprout in standard soil, in $35 \times 50 \times 10 \mathrm{~cm}$ flats maintained in the greenhouse for 4 to 5 weeks. The resulting seedlings were gently removed, washed under running tap water, and inoculated by dipping the roots in the inoculum suspension for approximately 15 s. The inoculated seedlings were transplanted immediately into standard soil. In both cases, control sets or seedlings were dipped into sterile, distilled water containing $0.5 \%$ agar.

For the infested soil method, the stock suspension of microsclerotia was adjusted to appropriate concentrations by diluting with sterile, distilled water, and the resulting suspensions were used to saturate (1:5, $\mathrm{vol} / \mathrm{vol}$ ) sterile silica sand (U.S Silica, Ottawa, IL). For the control treatment, sand was saturated with sterile, distilled water. The saturated sand was air dried at room temperature for 3 days, and mixed with standard soil $(1: 18.16$, vol/wt) to achieve the desired inoculum concentrations. Surface-sterilized sets were then planted in the resulting soil mixtures.

Disease evaluation. After inoculation or planting in infested soil, plants were grown in the greenhouse for approximately 2 months at 25 to $30^{\circ} \mathrm{C}$, under natural light supplemented with a combination of 1,000 $\mathrm{W}$, high-pressure sodium- and mercuryvapor lamps, set to a 12 -h per day photoperiod. Soil moisture was maintained by watering daily. Plants were examined regularly and scored 1 or 0 , respectively, for the presence or absence of foliar symptoms of Verticillium wilt. 
Two months after inoculation or planting in infested soil, plants were harvested to recover the original set roots. These were washed under tap water, cross-sectioned, and scored for the severity of each of the root symptoms, i.e., pepper, peg, or rot. For each of these symptoms, ratings were based on a 0 to 3 scale, with $0=$ no symptoms, $1=$ trace to less than $10 \%$ of the root cross section with pepper, peg, or rot symptoms, $2=10$ to $50 \%$ of the root cross section with pepper, peg, or rot symptoms, and $3=$ more than $50 \%$ of the root cross section with pepper, peg, or rot symptoms (Fig. 1). Since few roots had the peg or rot symptoms, only the ratings of pepper symptoms were considered for the computation of a wilt index, WId, similar to the "wilt score" described on cotton (31). In the current study, WId was obtained, for each plant, by adding numerical ratings of pepper and foliar symptoms. Thus, WId scores ranged from 0 to 4 , with $0=$ neither foliar nor pepper symptoms, and $4=$ the plant exhibited foliar symptoms and showed $>50 \%$ root discoloration.

Isolation of the pathogen. While plants were still growing in the greenhouse, attempts were made to isolate the pathogen from petioles of symptomatic leaves. When isolations were successful, the plant was considered infected and no further attempt was made to isolate the pathogen from roots. Otherwise, three small sections were excised from the root and petioles after harvest, and assayed for the recovery of the pathogen. In all cases, plant material was soaked for 30 to $60 \mathrm{~s}$ in $0.5 \% \mathrm{NaOCl}$, rinsed in three washes of sterile, distilled water, blotted dry, and aseptically pressed onto ethanol medium (6) in $100 \times 15 \mathrm{~mm}$ petri dishes. The plates were incubated at room temperature for 3 to 4 weeks, at which time $V$. dahliae was detected by the presence of microsclerotia. Dubious cases were evaluated further through microscopic examination. The experiment was repeated once.

Statistical analysis. The GLM and REG procedures of SAS (SAS Inc., Cary, NC) were used to analyze the data. Uninoculated controls were excluded from the analyses since symptoms specific to $V$. dahliae were not observed on these plants. The natural log transformation was applied to inoculum concentrations, and covariance analysis was performed on pooled data from the two trials. To check for the lack of fit of this reduced model, an analysis of variance was conducted on the same data based on a full model, in which inoculum concentration was treated as a quantitative variable. An $F$ test comparing the reduction in error sum of squares from the reduced to the full model was computed $(9,17)$.

Due to significant interactions between some of the factors involved in the covariance analysis of pooled data from the two trials, separate data from each trial, method, and cultivar combination were considered for regression analysis of WId on inoculum concentration. The goodness of fit of the adjusted models was evaluated by examining plots of standardized residuals against both predicted WId and inoculum concentration $(9,12)$.

\section{RESULTS}

For all the inoculation methods tested, foliar symptoms usually were observed first on 647A, the susceptible cultivar, treated with the two highest inoculum concentrations. The shortest (31 days) and longest (55 days) incubation periods, time from inoculation to the first foliar symptoms, were observed with the root dip and the oatseed methods, respectively. Incubation periods were intermediate (38 to 42 days) for the set dip and infested soil methods. Similarly, the highest $(68 \%)$ and lowest $(30 \%)$ incidences of foliar symptoms were obtained with the root dip and the oatseed methods, respectively. With the set dip and infested soil methods, incidence of foliar symptoms was 54 and $48 \%$, respectively.

About two-thirds (68\%) of the plants exhibiting foliar symptoms were of the susceptible cultivar, and only one-third (32\%) were of the resistant cultivar. The mean percent recovery of $V$. dahliae over the two trials was $37 \%$. However, the pathogen was reisolated more frequently

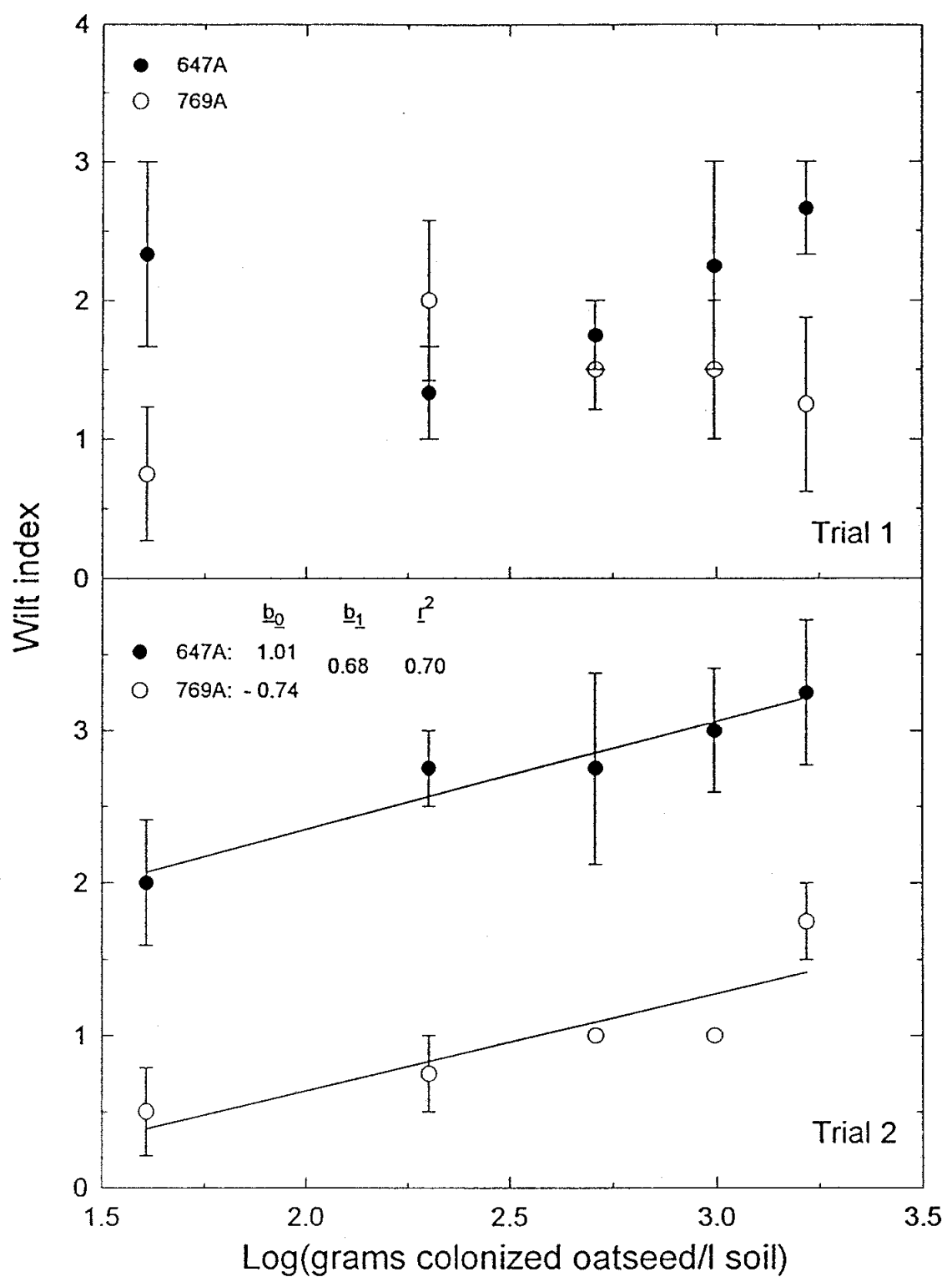

Fig. 3. Effect of inoculum concentrations of Verticillium dahliae on wilt index of two horseradish cultivars inoculated by colonized oatseed method. Each observation represents mean of three to four replications; vertical bars represent standard errors. Wilt index is on 0 to 4 scale, with $0=$ neither foliar nor pepper symptoms, and $4=$ plant exhibiting foliar symptoms and $>50 \%$ of root cross section with vascular discoloration. Trial 2: parameters are from covariance analysis of combined data for the two cultivars. 
from plants inoculated with colonized oatseeds (48\%) than from plants inoculated by other methods (34\% for each of the three other methods). Recovery of the pathogen also was higher on the susceptible (45\%) than on the resistant cultivar (30\%).

The covariance analysis of WId, based on data from the two trials, showed that all the inoculation methods tested were able to differentiate between the susceptible, 647A, and the resistant, 769A, cultivars. The root dip method gave the largest leastsquares means (lsmeans) difference between cultivars (Table 2). The highest $r^{2}$ and the lowest coefficient of variation also were obtained with the root dip inoculation method. With this method, significant lin- ear ID-disease (D) relationships were observed in 3 of the 4 cases examined, and the regression coefficients were similar (Fig. 2). Only in the first trial, on the susceptible cultivar, was the ID-D relationship not significant. In the second trial, ID-D relationships were significant for both cultivars. Since the cultivar-inoculum interaction was not significant, a pooled regression model (Fig. 2) was computed $(P=$ $0.0001)$, and the susceptible and the resistant cultivars were separated $(P=0.0001)$ on the basis of their WId lsmeans, 2.15 and 0.65 , respectively.

With the colonized oatseed method, no significant ID-D relationship was observed in the first trial, in contrast to the second

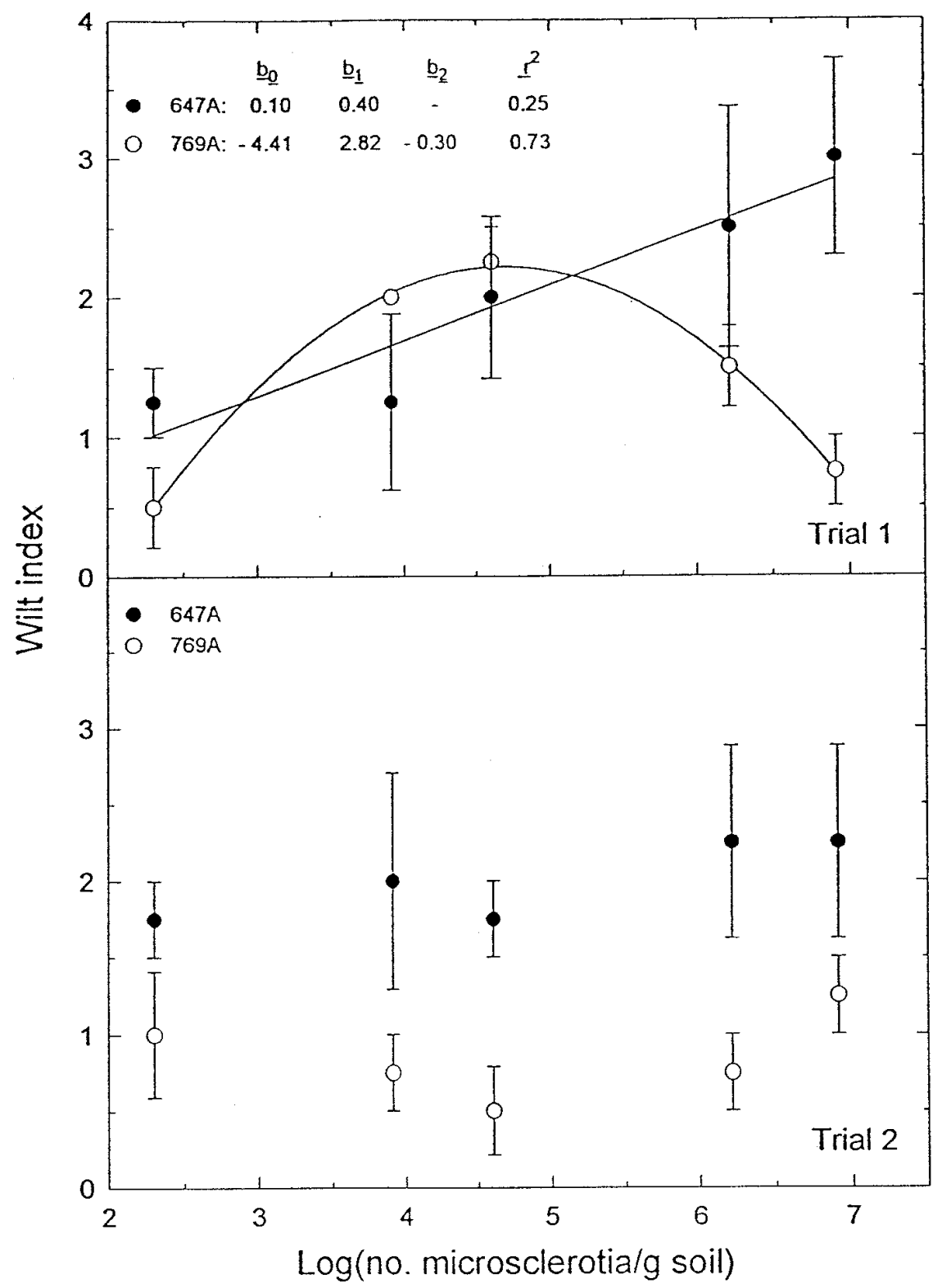

Fig. 4. Effect of inoculum concentrations of Verticillium dahliae on wilt index of two horseradish cultivars inoculated by infested soil method. Each observation represents mean of four replications; vertical bars represent standard errors. Wilt index is on 0 to 4 scale, with $0=$ neither foliar nor pepper symptoms, and $4=$ plant exhibiting foliar symptoms and $>50 \%$ of root cross section with vascular discoloration. trial, in which significant linear relationships were obtained for both cultivars (Fig. 3). The cultivar-inoculum interaction, however, was not significant in the second trial. Therefore, a unique regression coefficient (Fig. 3) was calculated $(P=0.0001)$, and WId lsmeans $(2.75$ for $647 \mathrm{~A}$ and 1.00 for $769 \mathrm{~A})$ were used to separate $(P=$ $0.0001)$ the two cultivars.

Significant ID-D relationships were observed in the first but not in the second trial with the infested soil method (Fig. 4). Nonetheless, the separation of cultivars $(P$ $<0.05)$ was better in the second than in the first trial, based on WId means of 2.00 and 0.85 for $647 \mathrm{~A}$ and $769 \mathrm{~A}$, respectively, in the second trial. During the first trial, there appeared to be a significant $(P=0.0685)$ cultivar-inoculum interaction, since the IDD relationship was linear $(P=0.0249)$ for the susceptible but curvilinear $(P=0.0001)$ for the resistant cultivars. Within the range of inoculum concentrations extending from 18 to 174 microsclerotia per $g$ of soil, the resistant cultivar had more disease than the susceptible, with a maximum at approximately 109 microsclerotia per $\mathrm{g}$ of soil (Fig. 4).

When plants were inoculated with the set dip method, the original sets were rotten in three out of four replicates of the susceptible cultivar challenged with 500 microsclerotia per ml, during the first trial. Therefore, this inoculum concentration was not considered during the regression analysis of the data on the susceptible cultivar. The equation derived from the remainder of the data, like the one obtained with the resistant cultivar, was significant only at the probability level $P=0.1200$. Nevertheless, a joint model (Fig. 5) was fitted ( $P$ $=0.0007)$, and the two cultivars were separated $(P=0.0007)$ on the basis of their WId lsmeans, 2.98 and 1.86, respectively, for 647A and 769A.

Significant linear ID-D relationships also were obtained with both cultivars inoculated by the set dip method, in the second trial. As with previous cases in which no cultivar-inoculum interaction was observed, data from the two cultivars were combined to fit $(P=0.0001)$ a pooled regression model (Fig. 5). Thereafter, lsmeans (2.00 for the susceptible and 0.80 for the resistant) were used to separate $(P=$ 0.0001 ) the two cultivars.

Overall, $r^{2}$ values ranged from 0.14 (regression analysis of data from the resistant cultivar inoculated by the set dip method in the first trial) to 0.73 (covariance analysis of combined data from the root dip method).

\section{DISCUSSION}

In this study, ID-D relationships were used to evaluate the effectiveness of four inoculation methods in identifying horseradish cultivars that are resistant to Verticillium wilt. In most cases, inoculum concentrations (log-transformed) had a signifi- 
cant effect on the WId; and ID-D relationships were linear except for the resistant cultivar with the infested soil method, in which a quadratic function best fit the data. The latter result is considered to be an experimental artifact (25).

Based on ID-D relationships, all inoculation methods revealed differences in the disease reaction of the susceptible and resistant cultivars. However, results obtained with the root dip method were more consistent, over the two trials, than were those obtained with the oatseed and infested soil methods. The root dip method also was reliable in that artifactual results, such as those obtained with the infested soil method, or rotting of sets as recorded with the set dip method, were not observed.

With the exception of the root dip method, pre-emergence rotting of sets that caused missing observations in the data analysis was a major concern, especially in the set dip inoculation method. In the root dip method, in which sets first develop secondary roots prior to challenging with the pathogen, this problem is easily circumvented by planting more sets than needed for the inoculation step. In this way, even if some sets fail to emerge, there are more than enough seedlings to inoculate, which also allows selection of plants of approximately the same growth stage for inoculation.

Another advantage of the root dip method lies in the fact that the inoculum is brought directly into contact with the host roots. This is not the case with either colonized oatseed or infested soil methods, which fall within Baker's "nonmotile inoculum-moving infection court" model (7). The model predicts that a large proportion of the inoculum remains dormant in the soil until activated by growing root tips. This may explain the longer incubation periods observed with these two methods. Although many of the asymptomatic plants were actually infected by $V$. dahliae based on isolations, the infection rate might be too low to make these methods useful for screening purposes when rapid detection of the disease is desirable.

As mentioned earlier, significant ID-D relationships were observed in most of the cases considered in this study. However, there were instances in which no ID-D relationship could be identified. Similar results have been reported on other Verticillium hosts for which significant ID-D relationships were observed in some cases $(1,3-5,10,15,20,21,24)$, but not in others $(2,11,15,21)$. Furthermore, even when a significant relationship has been found, there is no agreement as to the form of this relationship. Some investigators reported linear ID-D relationships $(1,3,4,10,15)$, while others found that ID could be more appropriately related to $\mathrm{D}$ by linear logit (20), quadratic (24), or negative exponential (21) models.
On horseradish, Percich and Johnson (22) observed a positive correlation between the concentrations of $V$. dahliae inoculum (up to 50 microsclerotia per $\mathrm{g}$ of soil), and the percent infection of roots, crowns, petioles, and leaves. Our data indicate that ID is a poor predictor of Verticillium wilt of horseradish. Although significant relationships between WId and ID were obtained in most cases, only a relatively low amount of the total variation in WId could be accounted for by ID $\left(0.14 \leq r^{2} \leq 0.73\right)$.

High levels of variability are commonly associated with studies on ID-D relationships involving Verticillium spp. The effects of fungistasis, soil moisture and/or aeration levels, soil nitrogen content, air temperature, changes in the pathogen population, differences in the physiology of individual plants, and the complex interactions with others microorganisms, e.g., Pratylenchus spp., are among the many factors most frequently proposed as possible explanations for poor ID-D relationships $(1,2,20,21,24)$. Nicot and Rouse (20) also incriminated the small number of samples usually involved in these studies. This argument is supported in the current study by the fact that substantial improvements in the $r^{2}$ were obtained each time observations from the two cultivars were combined.

Results obtained with the most dependable of the inoculation methods tested in

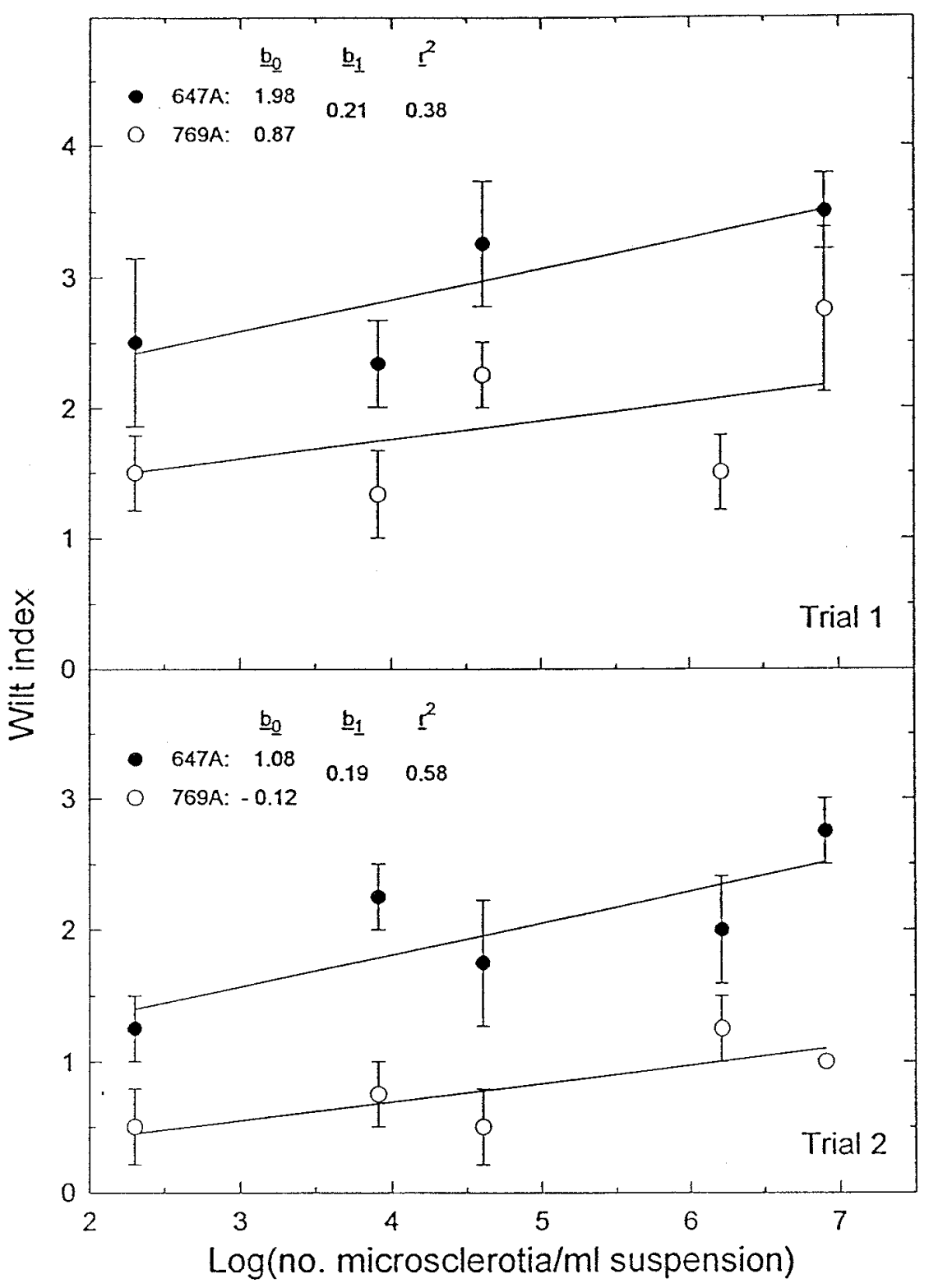

Fig. 5. Effect of inoculum concentrations of Verticillium dahliae on wilt index of two horseradish cultivars inoculated by set dip method. Each observation represents the mean of three to four replications; vertical bars represent standard errors. Wilt index is on 0 to 4 scale, with $0=$ neither foliar nor pepper symptoms, and $4=$ plant exhibiting foliar symptoms and $>50 \%$ of root cross section with vascular discoloration. For each trial, parameters are from covariance analysis of combined data for the two cultivars. 
the present study, the root dip method, suggest that ID is not (at least over the range tested) a critical factor in evaluating horseradish cultivars for resistance to $V$. dahliae. Differences in disease reaction would still have been detected even if the comparison was made between the susceptible cultivar at the lowest and the resistant cultivar at the highest inoculum concentrations (Fig. 2). Therefore, a natural and practical inoculum concentration, $100 \mathrm{mi}-$ crosclerotia per $\mathrm{ml}$, should be adequate for the screening of horseradish cultivars for resistance to $V$. dahliae, using the root dip method.

\section{ACKNOWLEDGMENTS}

The first author was supported by a fellowship from the Cameroon Institute of Agronomic Research and the World Bank. Statistical assistance was provided by G. Z. Gertner, Professor of Biometrics, Department of Natural Resources and Environmental Sciences, and C. A. Smyth, Statistical Laboratory, Department of Crop Sciences. We thank J. K. Pataky and W. L. Pedersen, Department of Crop Sciences, and the two anonymous reviewers for their helpful suggestions during the preparation of the manuscript.

\section{LITERATURE CITED}

1. Ashworth, L. J., Jr., Huisman, O. C., Grogan, R. G., and Harper, D. M. 1976. Copper-induced fungistasis of microsclerotia of Verticillium albo-atrum and its influence on infection of cotton in the field. Phytopathology 66: 970-977.

2. Ashworth, L. J., Jr., Huisman, O. C., Harper, D. M., and Stromberg, L. K. 1979. Verticillium wilt disease of tomato: Influence of inoculum density and root extension upon disease severity. Phytopathology 69:490-492.

3. Ashworth, L. J., Jr., Huisman, O. C., Harper, D. M., Stromberg, L. K., and Bassett, D. M. 1979. Verticillium wilt disease of cotton: Influence of inoculum density in the field. Phytopathology 69:483-489.

4. Ashworth, L. J., Jr., McCutcheon, O. D., and George, A. G. 1972. Verticillium albo-atrum: The quantitative relationship between inoculum density and infection of cotton. Phytopathology 62:901-903.

5. Ashworth, L. J., Jr., and Zimmerman, G. 1976. Verticillium wilt of the pistachio nut tree: Occurrence in California and control by soil fumigation. Phytopathology 66:14491451.
6. Ausher, R., Katan, J., and Ovadia, S. 1975. An improved selective medium for the isolation of Verticillium dahliae. Phytoparasitica 3: 133-137.

7. Baker, R., Maurer, C. L., and Maurer, R. A. 1967. Ecology of plant pathogens in soil. VII. Mathematical models and inoculum density. Phytopathology 57:662-666.

8. Chang, R. J., and Eastburn, D. M. 1994. Host range of Verticillium dahliae from horseradish and pathogenicity of strains. Plant Dis. 78: 503-506.

9. Chatterjee, S., and Price, B. 1991. Regression Analysis by Example. 2nd ed. John Wiley \& Sons, New York.

10. Davis, J. R., Pavek, J. J., and Corsini, D. L. 1983. A sensitive method for quantifying Verticillium dahliae colonization in plant tissue and evaluating resistance among potato genotypes. Phytopathology 73:1009-1014.

11. DeVay, J. E., Forrester, L. L., Garber, R. H., and Butterfield, E. J. 1974. Characteristics and concentration of propagules of Verticillium dahliae in air-dried field soils in relation to the prevalence of Verticillium wilt in cotton. Phytopathology 64:22-29.

12. Draper, N. R., and Smith, H. 1981. Applied Regression Analysis. 2nd ed. John Wiley \& Sons, New York.

13. Eastburn, D. M., and Chang, R. J. 1994. Verticillium dahliae: A causal agent of root discoloration of horseradish in Illinois. Plant Dis. 78:496-498.

14. Gerber, J. M., Doll, C. C., Simons, R. K., and Fillingim, K. E. 1983. Internal discoloration of horseradish - development of symptoms. Univ. Ill. Veg. Res. Rep. Hortic. Ser. 47:3437.

15. Grogan, R. G., Ioannou, N., Schneider, R. W., Sall, M. A., and Kimble, K. A. 1979. Verticillium wilt on resistant tomato cultivars in California: Virulence of isolates from plants and soil and relationship of inoculum density to disease incidence. Phytopathology 69:11761180.

16. Innes, N. L. 1992. Gene banks and their contribution to the breeding of disease resistant cultivars. Pages 23-31 in: Breeding for Disease Resistance. R. Johnson and G. J. Jellis, eds. Kluwer Academic Publishers, London.

17. Littell, R. C., Freund, R. J., and Spector, P. C. 1991. SAS System for Linear Models. 3rd ed. Sas Institute Inc., Cary, NC.

18. Maas, J. L., Draper, A. D., and Galletta, G. J. 1985. Inoculation methods for evaluating Verticillium wilt resistance in strawberry germplasm. HortScience 20:739-741.

19. Mueller, J. P., Percich, J. A., and Mitchell, J. E. 1982. Root deterioration associated with
Verticillium wilt of horseradish. Plant Dis. 66: 410-414.

20. Nicot, P. C., and Rouse, D. I. 1987. Relationship between soil inoculum density of Verticillium dahliae and systemic colonization of potato stems in commercial fields over time. Phytopathology 77:1346-1355.

21. Paplomatas, E. J., Bassett, D. M., Broome, J. C., and DeVay, J. E. 1992. Incidence of Verticillium wilt and yield losses of cotton cultivars (Gossypium hirsutum) based on soil inoculum density of Verticillium dahliae. Phytopathology 82:1417-1420.

22. Percich, J. A., and Johnson, D. R. 1990. A root rot complex of horseradish. Plant Dis. 74:391-393.

23. Platt, H. W., and Sanderson, J. B. 1987. Comparison of inoculation methods for field studies on varietal response to Verticillium wilt of potatoes. Am. Potato J. 64:87-92.

24. Pullman, G. S., and DeVay, J. E. 1982. Epidemiology of Verticillium wilt of cotton: A relationship between inoculum density and disease progression. Phytopathology 72:549554.

25. Vanderplank, J. E. 1975. Principles of Plant Infection. Academic Press, New York.

26. Virgin, W. J., and Maloit, J. C. 1947. The use of the seedling inoculation technique for testing tomatoes for resistance to Verticillium wilt. (Abstr.) Phytopathology 37:22-23.

27. Walker, J. C. 1975. Breeding for resistance to soil-borne pathogens. Pages 164-165 in: Biology and control of soil-borne plant pathogens, 3rd international symposium, 1973. G W. Bruehl, ed. American Phytopathological Society, St. Paul, MN.

28. Wiles, A. B. 1952. A seedling inoculation technique for testing cotton varieties for resistance to Verticillium wilt. (Abstr.) Phytopathology 42:288.

29. Wiles, A B 1960. Evaluation of cotton strains and progenies for resistance to Verticillium wilt. Plant Dis. Rep. 44:419-422.

30. Wilhelm, S. 1955. Longevity of the Verticillium wilt fungus in the laboratory and field. Phytopathology 45:180-181.

31. Wilhelm, S., Sagen, J. E., and Tietz, H. 1974. Resistance to Verticillium wilt in cotton Sources, techniques of identification, resistance trends, and the resistance potential of multiline cultivars. Phytopathology 64:924931.

32. Williams, P. H. 1989. Screening for resistance to diseases. Pages 335-352 in: The Use of Plant Genetic Resources. A. H. D. Brown, O. H. Frankel, D. R. Marshall, and J. T. Williams, eds. Cambridge University Press, New York. 